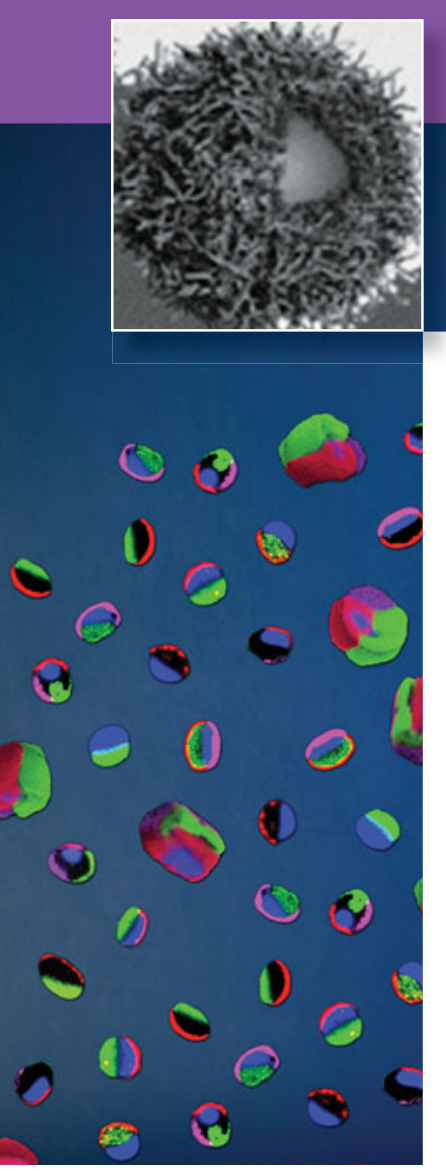

\title{
Organic nanoparticles for drug delivery and imaging
}

\author{
Samir Mitragotri and Patrick Stayton, Guest Editors
}

\begin{abstract}
Nanotechnology has had a huge impact on the development of therapeutics over the last couple of decades. To date, a large number of organic nanoparticles have been developed to encapsulate and deliver therapeutic and imaging agents. These nanoparticles have allowed encapsulation and targeted release of drugs. A few nanoparticle-based drugs are already being used in patients, and several others are making excellent progress toward clinical translation. The strong pipeline of therapeutic nanoparticles is fueled by advances in novel materials and design features, new applications, and a better understanding of fundamental hurdles that limit the utility of nanoparticles. The articles in this issue of MRS Bulletin are focused on some of the significant recent advances in the use of organic nanoparticles for drug delivery and imaging.
\end{abstract}

\section{Introduction}

Organic nanoparticles prepared from various materials, including polymers and lipids, have found exciting applications in therapeutic delivery and imaging (Figure 1). ${ }^{1}$ Nanoparticles offer distinct advantages over free drugs, including encapsulation, sustained or triggered release, and targeting to diseased sites. Encapsulation avoids exposure of drugs to off-target tissues. This provides a particular advantage for toxic drugs such as chemotherapeutics, where off-target toxicity is a key hurdle. Controlled release kinetics is also a key issue in drug delivery since pharmacokinetics often has a direct influence on therapeutic efficacy and toxicity. By choosing appropriate materials and design features, drugs can be delivered in a sustained or triggered manner. The ability of nanoparticles to target diseased tissues is perhaps their most appealing feature. Targeting has the potential to reduce off-target effects and enhance accumulation at the diseased site.

To date, tens of thousands of publications have reported various aspects of nanoparticle-mediated drug delivery. These include reports on novel materials and nanoparticle design, new applications of nanoparticles, and a better understanding of fundamental hurdles that limit the utility of nanoparticles. Advances in nanoparticle design and understanding have also led to new products, although the rate of conversion of scientific advances into commercial products has been alarmingly low. While only a handful of nanoparticle therapies are commercially available, a large number are in the pipeline at various stages of development.

This issue of MRS Bulletin is focused on some of the important recent advances in the use of organic nanoparticles for drug delivery and imaging.

\section{Design consideration for nanoparticles}

The design of nanoparticles, characterized by their material composition, size, shape, flexibility, and surface properties, essentially dictates their therapeutic outcome. A variety of materials have been used to synthesize nanoparticles, although lipids and polymers continue to be among the leading choices. Various synthetic polymers, including poly(lactic-co-glycolic) acid (PLGA), polyanhydrides, and dextrans, as well as natural polymers, including elastin-like polypeptides, have been used for preparing nanoparticles. ${ }^{2,3}$ The utility of these materials is determined by their toxicity, manufacturability, and compatibility with the drug. Hydrophobic drugs, which include many chemotherapeutic agents, can be easily encapsulated in hydrophobic polymers such as PLGA, whereas encapsulation of peptides and proteins in nanoparticles requires stringent considerations due to their susceptibility for unfolding, denaturation, and degradation. Polymer particles of various sizes from a few tens of nanometers to several micrometers have been synthesized and used for drug delivery. Many methods of nanoparticle synthesis have also been scaled-up: emulsification and 

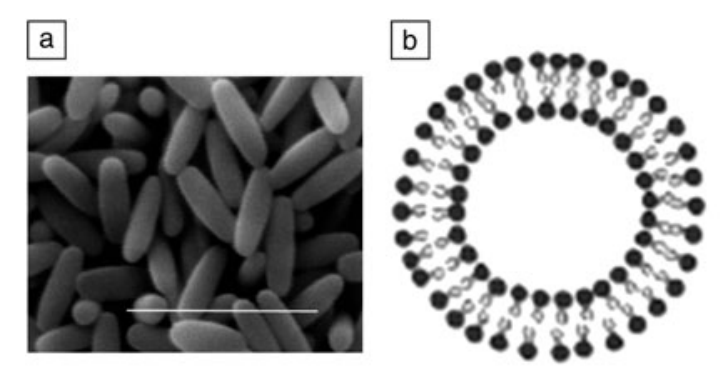

d

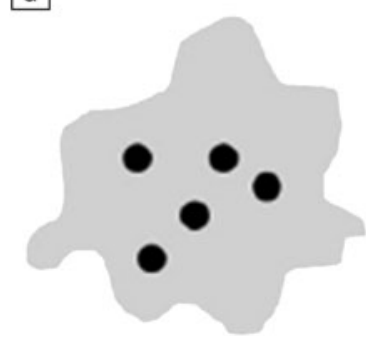

e
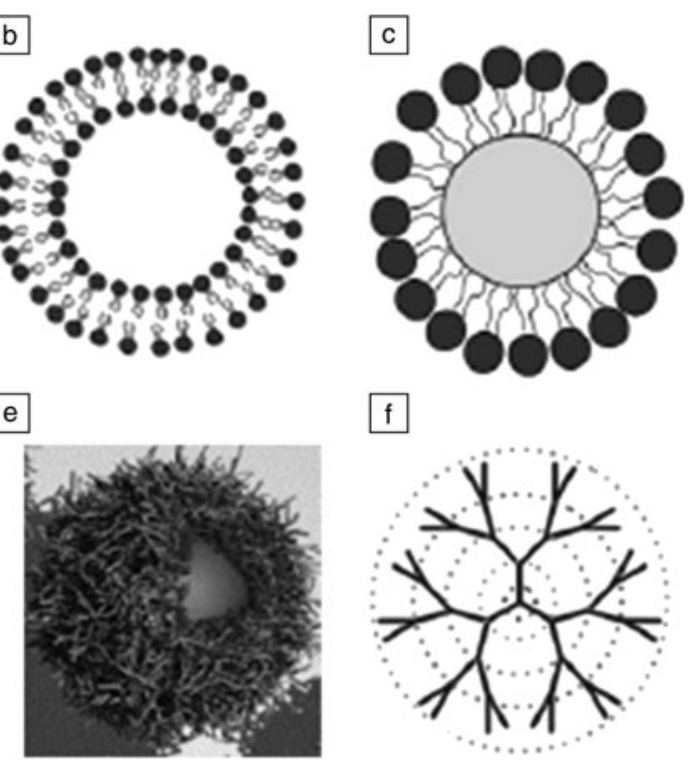

f

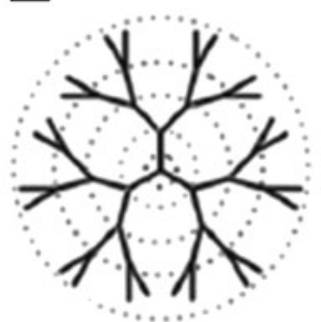

Figure 1. Examples of organic material-based carriers for drug delivery: (a) rod-shaped polymer particles (scale bar: $1 \mu \mathrm{m}$ ) (courtesy of Aaron Anselmo), (b) liposomes, (c) solidlipid nanoparticles, (d) protein-based nanoparticles (drug bound to protein), (e) layer-by-layer capsules, ${ }^{39}$ and (f) dendrimers. ${ }^{40}$ Reprinted with permission from Reference 38. () 2009 Wiley.

spray-drying, to name a few. Recently, methods have also been developed to synthesize particles of various shapes. ${ }^{4}$ These methods build on recent studies that show that non-spherical particles offer unique advantages in drug delivery. Specifically, rod-shaped nanoparticles have shown increased circulation ${ }^{5}$ and increased targeting, ${ }^{6}$ both of which are highly desired attributes in therapeutic nanoparticles. Methods such as particle replication in non-wetting templates (PRINT) have been developed to produce non-spherical particles in large quantities. ${ }^{7}$

Polymer nanoparticles also provide opportunities for triggered release. These systems allow additional control over targeting and further reduce systemic toxicity. Specifically, nanoparticles can be designed to prevent drug release altogether unless exposed to the trigger. Such triggers can be physically focused at the target site, thus allowing for increased targeting. Ultrasound and optical-based triggers have been used for this purpose. ${ }^{8,9}$ Triggers that make use of biomolecules present naturally at the diseased site (e.g., pH) have also been employed. ${ }^{10}$

Lipid-based systems (liposomes) also continue to be a popular choice for synthesizing nanoparticles. Lipids provide the advantages of biocompatibility and ease of encapsulation. They, however, are more fragile compared to polymeric nanoparticles. Liposome-encapsulated doxorubicin (Doxil) is the first example of successful use of nanoparticles for drug delivery, which is used to treat ovarian cancer, multiple myeloma, and AIDS-related Kaposi's sacrcoma. ${ }^{11}$ Block copolymers have also been used extensively for synthesis of nanoparticles. ${ }^{12}$ By controlling the lengths of the hydrophilic and hydrophobic components of block co-polymers, the size and shape of the nanoparticles can be controlled. ${ }^{12}$ Furthermore, the choice of the block co-polymer can also be used to control the degradation and release rate.

While polymer or lipid particles continue to be popularly used for drug delivery, the need for more advanced particles has arisen out of the complexity associated with encapsulation of multiple actives (Figure 2). Specifically, nanoparticles are increasingly being developed for theranostic applications where therapeutic and diagnostic agents are simultaneously encapsulated in a single nanoparticle. Given the significant differences in the physicochemical properties of these agents, their simultaneous encapsulation in a single nanoparticle is challenging. In addition, combination therapies, which are also becoming increasingly important, require encapsulation and release of multiple drugs, ideally with independently controlled release kinetics. Multi-compartmental particles are being developed to address this challenge where a single particle is composed of different compartments, each being made up of distinct drug/polymer combinations. ${ }^{13}$

While recent advances in the design and discovery of materials have led to several innovative nanoparticles, several challenges remain. Precise control of the drug release at the desired site still remains an unmet need. While triggered release systems offer a potential solution to this challenge, obtaining a perfect trigger is often a challenge. Many of the physical triggers such as lasers have limited tissue penetration. Controlling the intracellular fate of nanoparticles is another challenge. Most nanoparticles are internalized by cells via endocytosis, the process where nanoparticles bind to receptors and are internalized in membrane-vesicles, and are trapped in the lysosomes. Several nanoparticles have been designed to promote endosomal escape; however, their success remains limited. Further developments in this field are hindered by the inability to precisely monitor processes involved in endosomal escape. Accordingly, methods to promote better understanding of this phenomenon will open further avenues for future developments.

\section{Circulation of nanoparticles}

Controlling the fate of nanoparticles in the body is a major hurdle for targeted nanoparticles. Nanoparticles administered in the body have to travel through complex physiological hurdles before they arrive at their target. Non-specific clearance by the immune system, specifically, by the macrophages in the liver and the spleen, is a major challenge. Macrophages of the liver and spleen are very effective in the removal of nanoparticles from the body. In fact, a majority of the injected nanoparticle dose often ends up in the liver and spleen. ${ }^{12,14,15}$ Clearance of particles depends on their size. Larger particles 


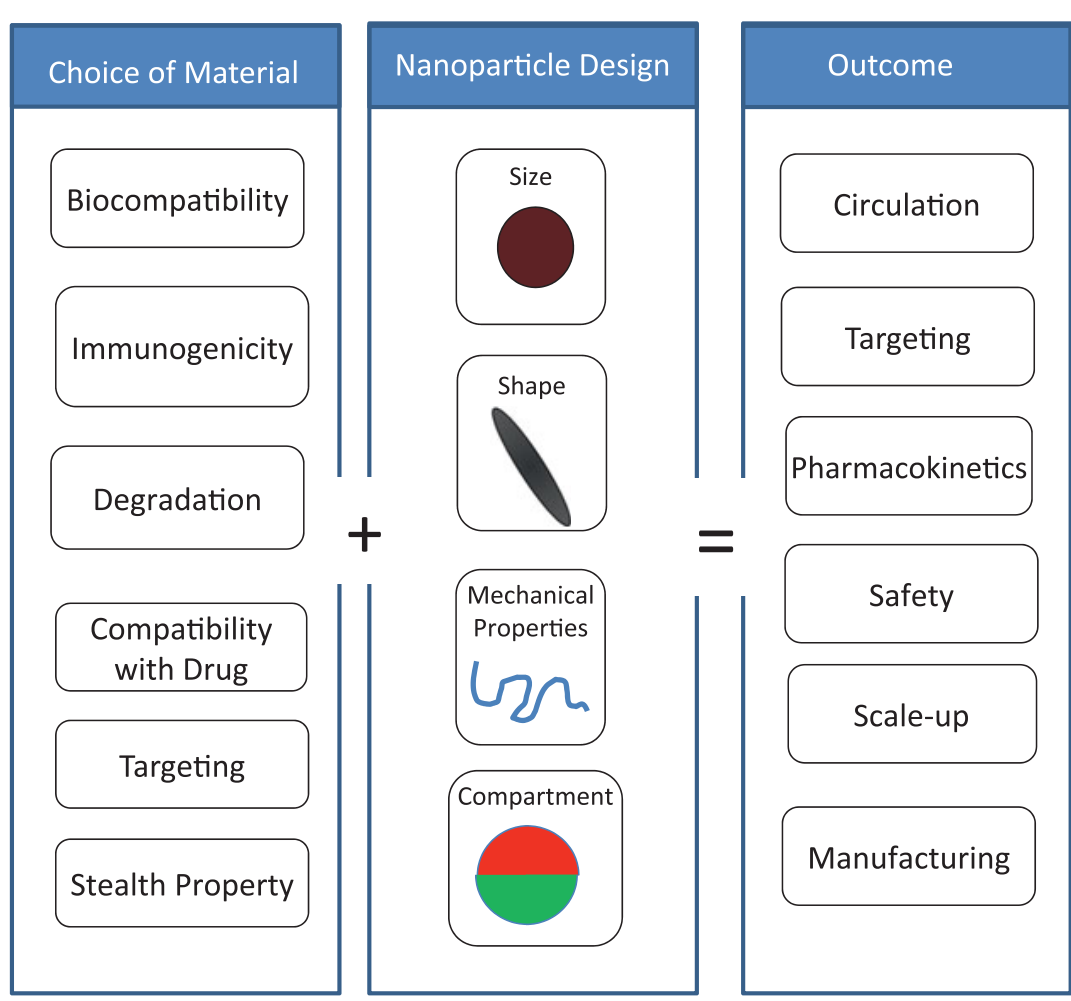

Figure 2. Design parameters for nanoparticles. The choice of material impacts various properties, including drug encapsulation, immunogenecity, and targeting. At the same time, the design of nanoparticles, such as size, shape, flexibility, and compartmentalization will also impact nanoparticle performance. These two attributes (choice of material and nanoparticle design) collectively determine the therapeutic outcome. particles with distinct morphologies ${ }^{20,21}$ and mechanical properties ${ }^{12,22}$ have also been developed. Elongated particles exhibit reduced macrophage uptake and enhanced circulation times. ${ }^{5}$ The flexibility of nanoparticles has also been shown to enhance circulation times and targeted accumulation. ${ }^{12,22}$

In another strategy, nanoparticles are placed on the surface of red blood cells to reduce their immune clearance. ${ }^{23}$ Nanoparticles have also been encapsulated in autologous (patient's own) red blood cells to prolong the circulation. ${ }^{24}$ Cloaking the nanoparticle surface with a red blood cell membrane has also been shown to prolong their circulation. ${ }^{25}$

While the current methods for enhancing the circulation of nanoparticles have brought about substantial improvements, a number of challenges remain. Regardless of the surface modification, nanoparticles are still recognized and cleared by the immune system. The nanoparticles must remain in circulation for sufficiently long times such as to reach their target. The precise value of the optimal circulation time may vary depending on the application. The short circulation time of nanoparticles is in significant contrast to natural systems, such as red blood cells, which circulate for months. Thus, substantial potential exists to further improve the circulation time of nanoparticles. Another hurdle is the fact that the strategies are trapped in small capillaries, primarily in the lungs, which can potentially interfere with lung function. Particles less than $200 \mathrm{~nm}$ in size show improved circulation half-life compared to larger particles. However, nanoparticles without any surface-modification often accumulate in the liver and spleen, regardless of their size, within minutes to hours after intravenous injection. ${ }^{16}$

Modification of the nanoparticle surface with "stealth" polymers is often used to reduce their clearance by the immune system. Polyethylene glycol (PEG) is often used for this purpose. ${ }^{17}$ Other materials such as dextran, di-block copolymers, and albumin are also used as stealth polymers. While coating the surface with these materials delays particle clearance, it does not entirely avoid it. In fact, some of these polymers, in particular PEG, are known to induce immune recognition, which leads to accelerated clearance after multiple injections. ${ }^{18}$

Recently, there has been a shift in paradigm to avoid the immune clearance. Unlike the conventional approach of using hydrophilic polymers to minimize the interactions with the immune system, the new strategies are purposefully aimed at interacting with the immune system. Scientists have begun exploring the use of CD47, a self-marker protein that can be used to trick the immune system to consider a foreign nanoparticle as its own. ${ }^{19}$ Other innovative approaches such as the use of to prolong the circulation often conflict with those used for targeting and vice versa. Specifically, the immune-evasive strategies aimed at minimizing interactions with cells are in direct contradiction to targeting strategies that aim to enhance cellular interactions. Striking a balance between them represents an opportunity for further research.

\section{Targeting of nanoparticles}

Targeting is a key hurdle in drug delivery. A significant fraction of research in targeted drug delivery has been performed in the context of tumor targeting. Tumor vasculature exhibits higher permeability compared to vasculature in healthy tissues, ${ }^{26,27}$ an effect termed the enhanced permeation and retention (EPR) effect. Nanoparticles accumulate in tumors through the EPR effect depending on their size. Particles within a size range of 10-100 nm are likely to exhibit high tumor accumulation through the EPR effect, ${ }^{28}$ although micron-size particles have also been shown to exhibit EPR. The ability of nanoparticles to accumulate in the tumor also depends on their circulation time in blood. Particles that circulate for longer times are more likely to come in frequent contact with the tumor tissue and accumulate in tumors. Particle shape has also known to impact the EPR effect. Worm-shaped flexible micelles have been shown to exhibit higher tumor accumulation due to 
Table I. Advantages and challenges associated with principal nanoparticles' features.

\begin{tabular}{|c|c|c|}
\hline Nanoparticle Feature & Advantages & Challenges \\
\hline Polymer-based particles & $\begin{array}{l}\text { Numerous choices available, } \\
\text { excellent stability and storage }\end{array}$ & $\begin{array}{l}\text { Burst release, challenges in } \\
\text { encapsulation of proteins }\end{array}$ \\
\hline Lipid-based particles & $\begin{array}{l}\text { Excellent biocompatibility, } \\
\text { proven history of use }\end{array}$ & $\begin{array}{l}\text { Limited storage stability, challenges in } \\
\text { loading of a broad variety of drugs }\end{array}$ \\
\hline Protein-based particles & Functional building material & $\begin{array}{l}\text { Potential immunogenicity issues, } \\
\text { stability }\end{array}$ \\
\hline Stealth coatings (PEG) & $\begin{array}{l}\text { Proven stealth properties, } \\
\text { renders colloidal stability }\end{array}$ & $\begin{array}{l}\text { Potential immunogenicity, } \\
\text { compromises targeting }\end{array}$ \\
\hline $\begin{array}{l}\text { Targeting agents (antibodies, } \\
\text { peptides, aptamers) }\end{array}$ & $\begin{array}{l}\text { Increased accumulation in } \\
\text { targets }\end{array}$ & Compromises circulation \\
\hline Small size & $\begin{array}{l}\text { Increased circulation, enhanced } \\
\text { EPR effect }\end{array}$ & $\begin{array}{l}\text { Limited sustained release, increased } \\
\text { potential for aggregation }\end{array}$ \\
\hline Non-spherical shape & $\begin{array}{l}\text { Longer circulation, enhanced } \\
\text { targeting, increased specificity }\end{array}$ & $\begin{array}{l}\text { Potential for complex manufacturing } \\
\text { process }\end{array}$ \\
\hline Compartmentalization & $\begin{array}{l}\text { Simultaneous loading of multiple } \\
\text { drugs, independent control of } \\
\text { drug release }\end{array}$ & $\begin{array}{l}\text { Potential for complex manufacturing } \\
\text { process }\end{array}$ \\
\hline
\end{tabular}

Note: PEG, poly(ethylene glycol); EPR, enhanced permeability and retention.

EPR compared to their spherical counterpart, likely due to their longer circulation times. ${ }^{12}$ In another study, discoidal particles showed shape-dependent EPR-mediated accumulation; discoidal-shaped micron-sized particles exhibited higher accumulation. ${ }^{29}$

Nanoparticles have been targeted to various tissues, including cancer cells, through modification of their surface with various targeting ligands, including peptides,$^{30}$ aptamers, ${ }^{1}$ and antibodies. ${ }^{31}$ Detailed discussions of ligand-mediated nanoparticle targeting can be found in several reviews. ${ }^{32}$ Peptides have also been identified to enhance tumor penetration. Specifically, peptides with the motif $\mathrm{R} / \mathrm{KXXR} / \mathrm{K}$ bind to neuropilin-1, a co-receptor for vascular endothelial growth factor, and lead to enhanced cellular entry. ${ }^{33}$ This peptide represents a potential biomimetic strategy to enter the cells and tissues. An example of such a peptide, internalizing RGD or iRGD, where RGD is arginine-glycine-aspartic acid, has been shown to deliver nanoparticles into tumors. ${ }^{34}$ Particle shape also affects the targeting efficiency. Recent studies have shown that rod-shaped nanoparticles exhibit better targeting compared to their spherical counterparts. ${ }^{6,35}$

While the field has clearly made strong strides in terms of targeted nanoparticles, several unmet needs remain. Even with the most advanced targeting strategy, intravascularly injected nanoparticles primarily accumulate in the liver and spleen. Clearly, better targeting strategies are desired. It is also increasingly recognized that therapeutic targets, especially tumors, are heterogeneous in terms of their biology. Accordingly, targeting strategies must take this heterogeneity into account. Such multi-targeting approaches are also likely to increase nanoparticle specificity toward the target. Several attempts have already been made to incorporate multiple ligands in a single nanoparticle. Future attempts should further develop such strategies. Strategies that make use of local, diseasespecific biology should also be studied. Examples of this include the use of specific tissue proteases (tissue-specific protein-degrading enzymes) for targeting purposes.

\section{Summary}

The benefits of organic nanoparticles in drug delivery and imaging have been clearly established. The field has witnessed impressive growth in fundamental understanding as well as novel applications of organic nanoparticles. Several innovative themes have recently emerged to guide the design of future nanoparticlebased therapies. Benefits and limitations of various features of nanoparticles must be taken into account while designing the future generation of therapeutic nanomaterials (Table I). These include the use of particle morphology and flexibility to control their in vivo behavior, the development of compartmentalized nanoparticles for encapsulating multiple agents, and the use of biomimicry and strategies for enhanced endosomal escape. ${ }^{36,37}$

The articles in this issue of MRS Bulletin highlight some of the recent advances in the field. Pearson et al. discuss the fundamental hurdles in nanoparticle interactions with cells and tissues that underline their success. They describe how physical properties of nanoparticles impact their key outcome, including targeting and deep tumor penetration. Yhee et al. describe the impact of materials on nanoparticle delivery. They discuss advantages of various choices and their use for cancer treatment. Rahmani and Lahann report the use of multicompartmental particles for drug delivery. Finally, Sengupta describes the latest advances in the design of nanoparticles for cancer treatment. He also discusses various considerations for nanoparticle design. Collectively, these reports highlight some of the most exciting advances in the field and outline future challenges. These opportunities as well as challenges provide an inspiration to a broad range of scientists engaged in a variety of fields, including materials, biology, and engineering, to discover and develop the next generation of drug delivery carriers.

\section{References}

1. O. Farokhzad, J. Cheng, B. Teply, I. Sherifi, S. Jon, P. Kantoff, J. Richie, R. Langer, Proc. Natl. Acad. Sci. U.S.A. 103 (16), 6315 (2006).

2. S.R. Macewan, A. Chilkoti, Nano Lett. 12 (6), 3322 (2012).

3. M.S. Muthu, M.K. Rawat, A. Mishra, S. Singh, Nanomedicine 5 (3), 323 (2009)

4. J.A. Champion, Y.K. Katare, S. Mitragotri, J. Control. Release 121 (1-2), 3 (2007). 
5. S. Muro, C. Garnacho, J. Champion, J. Leferovich, C. Gajewski, E. Schuchman, S. Mitragotri, V. Muzykantov, Mol. Ther. 16 (8), 1450 (2008).

6. S. Barua, J.W. Yoo, P. Kolhar, A. Wakankar, Y.R. Gokarn, S. Mitragotri, Proc. Natl. Acad. Sci. U.S.A. 110 (9), 3270 (2013)

7. L.E. Euliss, J.A. DuPont, S. Gratton, J. DeSimone, J., Chem. Soc. Rev. 35 (11), 1095 (2006)

8. J.F. Lovell, E. Huynh, T.D. MacDonald, Q. Lin, G. Zheng, Nanomedicine (London) 6 (7), 1156 (2011).

9. J.G. Morton, E.S. Day, N.J. Halas, J.L. West, Methods Mol. Biol. 624, 101 (2010).

10. K.C. Remant Bahadur, B. Thapa, P. Xu, Mol. Pharmaceutics 9 (9), 2719 (2012)

11. Y. Barenholz, J. Control. Release 160 (2), 117 (2012)

12. Y. Geng, P. Dalhaimer, S. Cai, R. Tsai, M. Tewari, T. Minko, D. Discher, Nature 2, 249 (2007).

13. K.J. Lee, S. Hwang, J. Yoon, S. Bhaskar, T.H. Park, J. Lahann, Macromol. Rapid Commun. 32 (5), 431 (2011)

14. Z. Liu, C. Davis, W. Cai, L. He, X. Chen, H. Dai, Proc. Natl. Acad. Sci. U.S.A. 105 (5), 1410 (2008).

15. A. Ayyagari, X. Zhang, K. Ghaghada, A. Annapragada, X. Hu, R. Bellamkonda, Magn. Reson. Med. 55 (5), 1023 (2006).

16. V.P. Torchilin, AAPS J. 9 (2), E128 (2007).

17. S.M. Moghimi, A.C. Hunter, J.C. Murray, Pharmacol. Rev. 53 (2), 283 (2001). 18. T. Ishida, M. Ichihara, X. Wang, K. Yamamoto, J. Kimura, E. Majima, H. Kiwada, J. Control. Release 112 (1), 15 (2006).

19. P. Oldenborg, A. Zheleznyak, Y. Fang, C. Lagenaur, H. Gresham, F. Lindberg, Science 288 (5473), 2051 (2000).

20. J. Champion, Y. Katare, S. Mitragotri, Proc. Natl. Acad. Sci. U.S.A. 104 (29), 11901 (2007).
21. D. Dendukuri, D. Pregibon, J. Collins, T. Hatton, P. Doyle, Nat. Mater. 5 (5), 365 (2006).

22. K.A. Beningo, Y. Wang, J. Cell Sci. 115 (4), 849 (2002).

23. E. Chambers, S. Mitragotri, Exp. Biol. Med. 232 (7), 958 (2007).

24. A. Antonelli, C. Sfara, L. Mosca, E. Manuali, M. Magnani, J Nanosci. Nanotechnol. 8 (5), 2270 (2008).

25. C.M. Hu, L. Zhang, S. Aryal, C. Cheung, R.H. Fang, Proc. Natl. Acad. Sci. U.S.A. 108 (27), 10980 (2011).

26. H. Maeda, Bioconjug. Chem. 21 (5), 797 (2010)

27. H. Maeda, Y. Matsumura, Adv. Drug Deliv. Rev. 63 (3), 129 (2011).

28. S. Acharya, S.K. Sahoo, Adv. Drug Deliv. Rev. 63 (3), 170 (2011).

29. P. Decuzzi, B. Godin, T. Tanaka, S.Y. Lee, C. Chiappini, X. Liu, M. Ferrari, J. Control. Release 141 (3), 320 (2010).

30. W. Arap, R. Pasqualini, E. Ruoslahti, Science 279 (5349), 377 (1998).

31. J. Sudimack, R. Lee, Adv. Drug Deliv. Rev. 41 (2), 147 (2000).

32. X. Ye, D. Yang, Cardiovasc. Hematol. Disord. Drug Targets 9 (3), 206 (2009).

33. T. Teesalu, K.N. Sugahara, V.R. Kotamraju, E. Ruoslahti, Proc. Natl. Acad. Sci. U.S.A. 106 (38), 16157 (2009).

34. K.N. Sugahara, Science 328 (5981), 1031 (2010).

35. P. Kolhar, A.C. Anselmo, V. Gupta, K. Pant, B. Prabhakarpandian, E. Ruoslahti, S. Mitragotri, Proc. Natl. Acad. Sci. U.S.A. 110 (26), 10753 (2013).

36. J.W. Yoo, D.J. Irvine, D.E. Discher, S. Mitragotri, Nat. Rev. Drug Discov. 10 (7), 521 (2011).

37. S. Mitragotri, J. Lahann, Nat. Mater. 8 (1), 15 (2009)

38. N. Doshi, S. Mitragotri, Adv. Funct. Mater. 19 (24), 3843 (2009).

39. A.P.R. Johnston, C. Cortez, A.S. Angelatos, F. Caruso, Curr. Opin. Colloid Interf. Sci. 11 (4), 203 (2006)

40. C.C. Lee, J.A. MacKay, J.M.J. Fréchet, F.C. Szoka, Nat. Biotechnol., 23 (12), $1517(2005)$
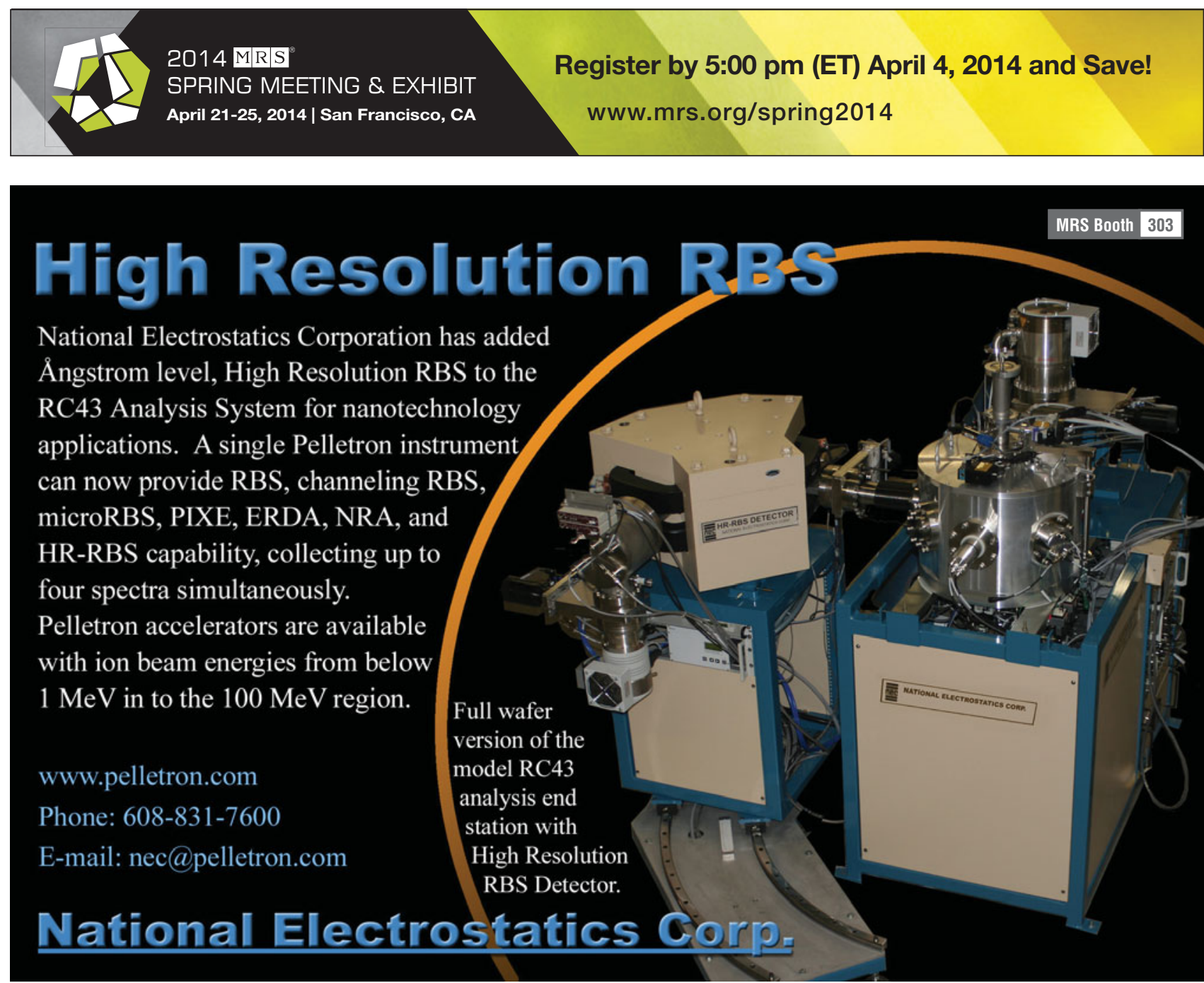